\title{
tic\&société
}

Vol. 9, N ${ }^{\circ}$ 1-2 | 1 er semestre 2015 - 2ème semestre 2015

Les religions au temps du numérique

\section{Des Powerpoints en chaînes.}

Formes et circulation des diaporamas chrétiens en Amérique latine

\section{Gustavo GOMEZ-MEJIA}

\section{(2) OpenEdition}

Journals

Electronic version

URL: http://journals.openedition.org/ticetsociete/1855

DOI: 10.4000/ticetsociete.1855

\section{Publisher}

Association ARTIC

\section{Electronic reference}

Gustavo GOMEZ-MEJIA, « Des Powerpoints en chaînes. », tic\&société [Online], Vol. 9, N 1-2 | 1er

semestre 2015 - 2ème semestre 2015, Online since 20 April 2019, connection on 30 April 2019. URL :

http://journals.openedition.org/ticetsociete/1855 ; DOI : 10.4000/ticetsociete.1855 
Des Powerpoints en chaînes.

Formes et circulation de diaporamas chrétiens en Amérique latine

Gustavo GOMEZ-MEJIA

Ëquipe Prim

Université François-Rabelais - IUT de Tours

29, rue du Pont-Volant, 37082 Tours Cedex 02

gustavo.gomez-mejia@univ-tours.fr 
Gustavo GOMEZ-MEJIA

Des Powerpoints en chaînes.

\section{Formes et circulation des diaporamas chrétiens en Amérique latine}

Gustavo GOMEZ-MEJIA est maître de conférences de sciences de l'information et de la communication à l'Université Francois-Rabelais (IUT de Tours) et membre de l'équipe Prim (Pratiques et ressources de l'information et des médiations). II a co-dirigé les ouvrages collectifs Persistances benjaminiennes (en collaboration avec O. Aïm, P. Boutin, J. Chervin et J.-F. Guennoc, Théorème 21, Presses Sorbonne nouvelle, 2014) et La médiatisation de l'évaluation (avec J. Bouchard, É. Candel et H. Cardy, Peter Lang, 2015). Ses recherches portent principalement sur les identités, les industries culturelles et les dispositifs du web contemporain.

Résumé : Le présent article s'intéresse aux formes et aux modes de circulation des Powerpoints comme supports numériques de signes religieux dans l'espace latino-américain. À partir d'un corpus de diaporamas distribués par courrier électronique, l'auteur analyse trois dimensions du phénomène des "chaînes religieuses ". D'une part, les caractéristiques que ces chaînes partagent avec des pratiques postales anciennes aujourd'hui reconfigurées par les messageries sur écran. D'autre part, les modalités d'inscription des imaginaires et textualités chrétiennes sous forme de présentations Powerpoint. Enfin, la manière dont ces diaporamas reflètent les paysages de croyances de l'Amérique latine contemporaine. Des approches à dominante sémiologique permettent de discerner l'épaisseur culturelle de ces objets ordinaires pour comprendre les mystères de leur circulation.

Mots-clés : religion, Internet, chrétienté, chaînes de courriels, Microsoft Powerpoint, diaporama, vernaculaire, Amérique latine.

Abstract: On PowerPoint chain letters. Forms and circulation of Christian slideshows in Latin America - This article focuses on the forms and circulation of Powerpoint slideshows as digital supports for religious signs in Latin American space. A corpus 
of presentations sent by e-mail allows the author to analyze three main dimensions of the "religious chain" phenomenon. Firstly, the characteristics that such chains share with older postal practices nowadays remediated by on-screen e-mail services. Secondly, the modes in which Christian texts and imageries are reshaped by Powerpoint formats. Lastly, the ways in which such slideshows reflect the evolving landscape of beliefs in Latin America. Semiotic approaches contribute to the comprehension of both the cultural complexities of these ordinary objects and their mysterious circulation.

Keywords: religion, Internet, Christianity, Microsoft Powerpoint, e-mail chains, vernacular, Latin America.

Resumen: Sobre los Powerpoints en cadenas. Formas y circulación de diapositivas cristianas en América Latina. - Este artículo aborda las formas y los modos de circulación de los formatos Powerpoint como soportes digitales de signos religiosos en el espacio latinoamericano. Con base en un corpus de presentaciones distribuidas por correo electrónico, el autor analiza tres dimensiones del fenómeno de las « cadenas religiosas ». Por un lado, las características que dichas cadenas comparten con prácticas postales antiguas hoy reconfiguradas por los buzones en pantalla. Por otra parte, los modos de inscripción de los imaginarios y textualidades cristianas formateadas como diapositivas de Powerpoint. Por último, la manera en la que tales presentaciones reflejan los paisajes de creencias de la contemporaneidad latinoamericana. A través de enfoques semiológicos se busca así entender la complejidad cultural de dichos objetos ordinarios y los misterios de su circulación.

Palabras clave: religión, Internet, cristiandad, cadenas de correos, Microsoft Powerpoint, vernáculo, América Latina. 
Les présentations Powerpoint occupent une place privilégiée parmi les dispositifs numériques que les populations mondiales, et en particulier latino-américaines, mobilisent au quotidien pour exprimer diverses nuances (catholiques, évangéliques, syncrétiques) d'une foi chrétienne. Par l'intermédiaire des "chaînes » de courriers électroniques que l'espagnol nomme " cadenas », un volume inestimable de diaporamas religieux attachés en pièce-jointe circule chaque jour entre les messageries électroniques de sujets plus ou moins croyants. Visiblement agacée par l'ampleur de cette pratique courante, une journaliste colombienne propose à ceux qui ne le connaissent pas une première définition (aux traits caricaturaux) du phénomène numérique des « cadenas » :

"Fondamentalement, ces chaînes ne sont rien d'autre que des fichiers joints sous divers formats de présentation dont les contenus sont extraits de la Bible; à leur lecture, on dit au destinataire qu'il est entré en grâce et que s'il fait suivre ces fichiers il recevra une bonne nouvelle, trouvera un emploi ou gagnera de l'argent; s'il ne le fait pas, il aura mauvaise fortune » (Acevedo Moreno, 2013).

Dans le cas spécifique des fichiers Powerpoint, une «chaîne religieuse " (cadena religiosa) se maintiendra ainsi en circulation tant que le visionnage du diaporama par un sujet destinataire sera suivi d'un renvoi du fichier reçu à d'autres contacts. L'enchaînement de messages propitiatoires et de gestes de propagation que chaque " chaîne " cristallise nous place au carrefour des relations qu'entretiennent les croyances traditionnelles et les techniques contemporaines, là même où deux questions fondamentales se posent pour le chercheur en communication: comment parvient-on à élaborer des textualités et des imaginaires religieux sous forme de Powerpoint? Quel sens pouvons-nous donner à cette pratique qui relie faire chaîne, faire suivre et faire croire ? De fait, cette appropriation culturelle du format de présentation mis au point par Microsoft nous interpelle comme objet d'étude pour deux raisons principales. D'une part, elle prête le langage d'un support informatisé stable à un faisceau de croyances chrétiennes en Amérique latine. D'autre part, elle anticipe les conditions de circulation de ces fichiers tenus pour dépositaires d'un message religieux.

Ce questionnement communicationnel nous invite à analyser les « chaînes » de Powerpoints chrétiens dans un mouvement qui confronte les «matrices culturelles» issues de la colonisation catholique en Amérique latine au devenir des "formats industriels" de l'informatique en réseau (MartinBarbero, 2003, p.XVI). Pour concrétiser ce projet sur le plan 
méthodologique, nous avons procédé à la constitution d'un corpus principal de diaporamas religieux, rédigés en langue espagnole et circulant par courriel au format Microsoft Powerpoint. Comment se saisir de ces matériaux anecdotiques et « non-institutionnels » que les folkloristes d'Internet nomment «vernaculaires»? (Howard, 2008). Dans une logique ellemême réticulaire, entre les mois d'avril et mai 2014 , nous avons sollicité plusieurs proches d'origine latino-américaine et leurs relations (en Colombie, au Brésil, en Argentine, mais aussi en Floride ou en Italie) afin qu'ils nous retransmettent quelques «Powerpoints religiosos » pour une étude, sans leur donner plus de précisions. Au total, ces informateurs volontaires nous ont permis de recueillir par retour de courriel une douzaine de diaporamas et courriels respectifs qui sont au fondement empirique de cette recherche.

Quelques impressions spontanées des informateurs, doublées de recherches documentaires sur les «chaînes" dans le monde et en Amérique latine, complètent les ressources de cette étude que nous déroulerons en trois temps complémentaires, sensibles aux "versants logistiques et sémiotiques » de cette pratique «triviale » (Jeanneret, 2009). Notre objectif est de comprendre comment se négocie cette conversion des cadres standardisés du courrier électronique et du diaporama à un usage spécifiquement religieux dans le contexte latino-américain. Dans cet état d'esprit, un premier mouvement historique et morphologique s'intéressera aux traits constitutifs d'une pratique religieuse de la "chaîne " reconfigurée sous forme de courriel. Un deuxième mouvement portera plus spécifiquement sur la teneur des croyances mises au format Powerpoint et sur les effets de sens qui motivent leur remise en circulation. Un troisième et dernier mouvement envisagera la question de la circulation des Powerpoints à l'aune de quelques tensions structurantes du paysage des croyances latino-américaines contemporaines.

\section{De la chaîne de lettres à celle de courriels : la mémoire d'une pratique religieuse reconfigurée}

$A$ contrario des projections « révolutionnaires » qui verraient dans la prolifération de «cadenas religiosas» des manifestations ultra-contemporaines d'une « cyber-religion » (Gil, 2006), la cristallisation des messages chrétiens sous forme de « courriers » nous renvoie à une longue histoire culturelle au cours de laquelle s'est décantée une «logique de l'usage » de la correspondance comme " chaîne » sous des auspices plus ou moins «magiques, religieux ou profanes » (Perriault, 1989 ; Bonnet \& Delestre, 1984; Rouquette, 1994, p.27). Ainsi, en 
amont de nos messageries électroniques modernes, certains analystes des pratiques postales ont pu documenter l'évolution historique des "chaînes de lettres" manuscrites puis imprimées. Définissant la « chain letter» comme une «lettre qui demande explicitement au destinataire de faire ou d'acheter des copies et de les distribuer », le folkloriste américain Daniel VanArsdale (1998) voit dans les «Lettres du Ciel » européennes (Himmelsbriefe) - «supposément écrites par Dieu lui-même ou par un agent divin » et remontant au $\mathrm{VI}^{\mathrm{e}}$ siècle - le principal «prédécesseur 》 des chaînes contemporaines (religieuses ou sécularisées) qui circulent de nos jours par courriel ${ }^{1}$. Cet héritage «apocryphe » des Himmelsbriefe hante une partie considérable des chaînes de courriels de notre corpus lorsqu'elles se donnent pour « objet » explicite le «Testament du Christ», une «Interview avec Dieu », une «Conversation de Jésus avec le Diable » ou même une "Prophétie de la Vierge de Fatima » suivie d'un "lisez et distribuez-la » en guise d'injonction directe à la répliquer ${ }^{2}$.

Bien évidemment, d'une chaîne à l'autre, des Himmelsbriefe en latin aux courriels latino-américains, les contextes techniques et géoculturels de ces phénomènes de matérialisation et remise en circulation d'un système de croyances chrétiennes varient amplement. Cependant, dans la mesure où les dispositifs du courriel «met[tent] en scène une situation qui a pour référent le courrier postal » (DesprèsLonnet et al., 2003), il semble pertinent d'établir des points de contact entre les principaux traits caractéristiques que les discours folkloristes prêtent aux anciennes chaînes de lettres religieuses et les modalités de cristallisation qu'ils adoptent dans le cadre sémio-technique des messageries électroniques. Comment se configurent à l'écran ces caractères “apocryphes » et « réplicatifs 》 (VanArsdale, 1998) des messages religieux qui semblent favorables à la projection des croyances et par conséquent à l'origine de l'effet de chaîne ?

En ce qui concerne le caractère « apocryphe » des chaînes religieuses, celui-ci renvoie à la fois à l'autorité et à l'auctorialité des croyances qui circulent par courriel : tant le testament, que l'interview ou la prophétie précités relèvent a priori de ce dont "l'Église ne reconnaît[rait] pas l'origine divine » et dont

\footnotetext{
${ }^{1}$ Les «Neuvaines » manuscrites et dactylographiées qu'étudie le psycho-sociologue Michel-Louis Rouquette, "reçues par voie anonyme " et où l'on demande de « recopier une lettre pendant 9 jours pour l'envoyer à 9 personnes différentes » pour obtenir des grâces des saints, pourraient être également considérées comme des ancêtres des chaînes contemporaines de courriels religieux (Rouquette, 1994, p.18).

${ }^{2}$ Respectivement "Testamento de Cristo", "Entrevista con Dios" "Jesús conversa con el diablo", "Profecías de la virgen de Fátima - léanla y distribúyanla", selon leurs titres originaux.
} 
"l'appartenance au canon des livres inspirés de la Bible semble douteuse ou erronée » (Trésor de la langue française). Ce même qualificatif qui fait le pont entre «ce qui est faussement attribué à une époque ou à un auteur » et l'étymologie d'apokryphos "secret, caché » (Calvino, 1998, p.206) peut concerner les réponses que d'autres chaînes prétendent apporter à des questions doctrinales : «Qu'y a-t-il au centre de la Bible?", "Définitions de religion et de spiritualité », «Où va-t-on quand on décède ? », "Pourquoi faire confiance à Jésus-Christ ? ${ }^{3}$. De manière générale, ces courriels restent apocryphes dans la mesure où ils prétendent émaner d'une source divine non attestée ou se prononcer sur des dogmes chrétiens sans incarner une autorité institutionnelle. Au sens technique du terme, ni le Christ, ni Dieu, ni la Vierge, ni une quelconque Église ne sont les «auteurs » de ces chaînes de courriels. En ce sens, "les chaînes constituent des procédés de communication et de mobilisation "parallèles", qui traduisent l'effet d'initiatives en marge de l'institution officielle ecclésiastique » (Rouquette, 1994, p.20). Cette part de « secret», ce décalage entre le message qui viendrait apparemment «d'en haut» et son anonyme scripteur réel, délivre la chaîne de courriels de toute figure unitaire de l'initiateur ou du garant énonciatif de la fidélité du message. À l'écran, le caractère apocryphe du message religieux favorise donc le fait qu'il soit « appropriable »: à défaut d'un auteur central (dont la teneur symbolique est néanmoins récupérée), le dispositif de messagerie valorise avant tout le rôle de «l'expéditeur» dont le nom est automatiquement affiché : «Rosa Victoria - Fwd: FW: Rv: Rv: TESTAMENTO DE CRISTO ».

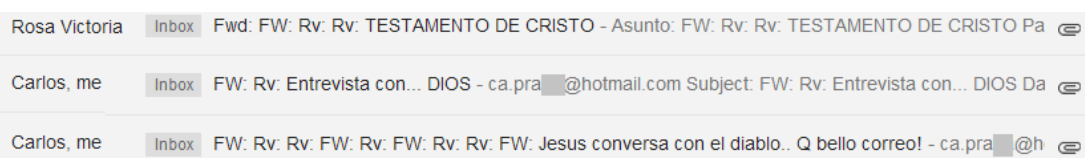

Fig. 1 : Affichage des chaînes religieuses sur la messagerie Gmail.

Entre la cooccurrence du nom propre de l'auteur du courriel et l'objet « apocryphe » qu'il semble s'approprier, les dispositifs de messagerie électronique nous signalent aussi avec leur nomenclature informatique l'autre grand trait constitutif des " chaînes religieuses » : leur caractère « réplicatif ». A l'écran,

\footnotetext{
${ }^{3}$ En espagnol : "Qué hay en el centro de la biblia?”, "definiciones de religión y de espiritualidad", "A donde va uno cuando fallece?", "Por qué confiar en Jesucristo?".
}

tic\&société - 9 (1-2), 2015 
les particules «FW: $R v: R v$ : » indiquent au « destinataire » qu'il s'agit littéralement du renvoi d'un renvoi d'un renvoi. Dans le champ «Objet » du courriel, ces accumulations systématiques des équivalents latino-américains du «TR: » français souvent suivies d'écritures en majuscules qui se veulent importantes, suggèrent au «destinataire » qu'à son tour il pourrait s'approprier l'objet " apocryphe » le temps d'un courriel et devenir "un maillon de la chaîne » de circulation des croyances religieuses. «Le fait d'ignorer leur origine n'invalide pas leur circulation », car « on peut toujours identifier [le nom de] la dernière personne qui a renvoyé la chaîne et il s'agit souvent d'une "connaissance" [du récepteur] " eștime l'anthropologue argentine Flora Delfino (2006, p.109). À en juger par ces marques de circulation que les messageries apposent à la chaîne par défaut, les Powerpoints de notre corpus sont parvenus à nos informateurs au bout de deux à trois renvois en moyenne. Or, lorsque les « expéditeurs" n'effacent pas le corps du courriel, nous avons accès à l'inventaire des " maillons de "connaissances" de la chaîne ». Ainsi, une traîne résiduelle de noms d'expéditeurs, adresses et mentions «forwarded message » nous apprend par exemple que la chaîne "Prophétie de la Vierge de Fatima - lisez et distribuez-la » en est déjà à son sixième renvoi et que chacun de ses « expéditeurs " incorpore une vingtaine de nouveaux « destinataires » susceptibles de la répliquer à leur tour.

Pris dans l'économie numérique de ce qui donne l'impression de se laisser répandre ou propager facilement - la spreadability de Jenkins (2009) - les Powerpoints des chaînes religieuses deviennent de véritables contenus d'énième main qui se laissent « adopter » par un « contact» proche. « Je sais bien que ces contenus ont des origines opaques, mais quand même, je forwarde », pourrait-on dire en paraphrasant la célèbre formule du psychanalyste Octave Mannoni, et par làmême rappeler que le propre du croyant est d' " être en état de savoir et de ne pas savoir » (Lambert, 2013). Savoir reconnaître une valeur au fichier apocryphe et le « faire suivre » en un geste à un "carnet d'adresses électronique » qui recense les noms d'autres « connaissances » croyantes. Savoir donc également que tous ces noms écrits ensemble dans le champ « destinataire » se reconnaissent mutuellement comme partageant une disposition ou un sentiment religieux commun. Ne pas savoir, en revanche, si les pièces-jointes transmises par courriel susciteront ces « adhésions provisoires 》 (Ibidem) qui transforment les destinataires en ré-expéditeurs et témoignent de l'efficacité du couple courriel + Powerpoint comme support du religieux. "Avant je recevais des courriels comme ça, mais je n'en ai plus reçu. Je crois que les gens me connaissent et savent que je ne vais pas les ouvrir et encore moins les 
renvoyer », expliquait un informateur sorti de ces circuits informels de mailings de croyants.

\section{Des diaporamas chrétiens: modes de production d'une religiosité au format Powerpoint}

Au-delà du cadre du courriel qui actualise la chaîne comme forme plus ou moins archaïque de mise en circulation des croyances, c'est du côté des pièces-jointes collectées qu'il faut se pencher pour comprendre la production d'une épaisseur religieuse à l'écran sous forme de fichiers Powerpoint. Au sens où l'entend David Douyère (2014), nous nous intéressons donc à « un type particulier d'image de piété, scénarisé, l'assemblage animé et interactif d'images fixes constitué par la présentation Powerpoint de méditation, que nous pourrions qualifier par voie de raccourci de "Powerpoint chrétien" ». Les pièces-jointes (attachments) des chaînes collectées nous montrent un spectre de variantes latino-américaines de ces diaporamas chrétiens que $D$. Douyère situe à juste titre « au croisement de la lecture spirituelle illustrée (prières, citations bibliques, images religieuses) et de la méditation guidée. " Pendant le temps du défilé des diapositives (slides) d'une présentation, les croyances chrétiennes se trouvent façonnées par «l'écriture plastique » des formats .ppt ou .pps. (Tardy \& Jeanneret, 2007, p.214), bien connue d'autres usages professionnels ou ludiques, qui «trace un chemin de lecture, illustrée et animée » (Douyère, 2011). Pour explorer ce chemin, nous pouvons déconstruire à l'aide d'un tableau synoptique les modes d'émergence de connotations religieuses dans la plasticité même des Powerpoints précités. Une grille sémiologique barthésienne (Barthes, 1964) peut s'attaquer à leur assemblage d'inscriptions et d'images, d'effets d'animation audiovisuelle, mais aussi aux séquences rhétoriques finales adressées aux sujets croyants censés devenir « maillons » de la chaîne en question. 
tic\&société - 9 (1-2), 2015

\begin{tabular}{|c|c|c|c|c|}
\hline $\begin{array}{l}\text { Fichier } \\
\text { Powerpoi } \\
\text { nt } \\
\end{array}$ & Messages linguistiques & $\begin{array}{l}\text { Messages } \\
\text { Iconiques }\end{array}$ & $\begin{array}{l}\text { Effets } \\
\text { dramatiques }\end{array}$ & $\begin{array}{l}\text { Adresse finale } \\
\text { au récepteur }\end{array}$ \\
\hline $\begin{array}{l}\text { Testament } \\
\text { o de } \\
\text { Cristo.pps } \\
\text { [20 diapos] }\end{array}$ & $\begin{array}{l}\text { - Énoncés à la } 1^{\text {ere }} \text { personne qui } \\
\text { pastichent l'incipit d'un testament : } \\
\text { « Je soussigné, Jésus de Nazareth, } \\
\text { voyant que mon heure approche... ». } \\
\text { - Inventaire hautement symbolique } \\
\text { commenté de ce qu'll lègue à } \\
\text { « l'humanité »: « l'étoile, la crèche, } \\
\text { mes sandales [...] le calice, la croix, } \\
\text { ma tunique [...] », mais aussi des } \\
\text { « attitudes »: « ma parole, la joie, } \\
\text { l'humilité, mon épaule, mon } \\
\text { pardon ». } \\
\text { - Déclaration finale : « Je vous ai } \\
\text { aimé à l'extrême et vous porte dans } \\
\text { mon cœur ». Signé : « Jésus ». }\end{array}$ & $\begin{array}{l}\text { - Photo de fleurs } \\
\text { blanches sur fond } \\
\text { noir [§1-12, 14, } \\
20] \\
\text { - Icônes de } \\
\text { Jésus : } \\
\text { hors-tombeau en } \\
\text { tunique blanche; } \\
\text { avec le Sagrado } \\
\text { Corazón (Sacré- } \\
\text { Cœur); } \\
\text { de profil ; } \\
\text { avec un ange ; } \\
\text { auréolé les bras } \\
\text { ouverts ; } \\
\text { [§13, 15-19]. }\end{array}$ & $\begin{array}{l}\text { - Fondu } \\
\text { filmique : titre } \\
\text { Testamento } \\
\text { de Cristo. } \\
\text { - Musique } \\
\text { « Winter Light } \\
\text { de Linda } \\
\text { Ronstadt » } \\
\text { (voix, } \\
\text { réverbération, } \\
\text { piano). } \\
\text { - Gif animé } \\
\text { de Jésus } \\
\text { bénissant } \\
\text { d'un signe de } \\
\text { croix [§ 19]. }\end{array}$ & $\begin{array}{l}\text { “Envoie ce courriel à autant de } \\
\text { contacts que tu en as. } \\
\text { Ainsi tu donneras à d'autres } \\
\text { l'opportunité de savoir que nous } \\
\text { n'avons jamais été seuls dans } \\
\text { les moments difficiles et qu'il y a } \\
\text { en Christ une nouvelle vie et } \\
\text { beaucoup de repos. } \\
\text { Maintenant apprécie l'amour de } \\
\text { Jésus dans ta vie et dans celle } \\
\text { de ta famille dans l'action de } \\
\text { renvoyer cette Bénédiction. » }\end{array}$ \\
\hline $\begin{array}{l}\text { Entrevista } \\
\text { con } \\
\text { Dios.pps } \\
\text { [13 diapos] }\end{array}$ & $\begin{array}{l}\text { - Récit onirique : « J'ai rêvé que } \\
\text { j'avais un entretien avec Dieu... - } \\
\text { Entrez SVP, dit Dieu. Alors c'est } \\
\text { vous qui aimeriez m'interviewer? ? } \\
\text { - Questions/réponses : sur la durée } \\
\text { de l'interview ( « mon temps est } \\
\text { éternel ») ; sur « ce qui le surprend } \\
\text { dans l'humanité » (rapports } \\
\text { paradoxaux âge adulte/enfance, } \\
\text { santé/argent, futur/présent, vie } \\
\text { mort) ; sur les « leçons de vie pour } \\
\text { ses enfants » (se laisser aimer, avoir } \\
\text { des proches autour, ne pas se } \\
\text { comparer, ne pas blesser, } \\
\text { pardonner, relativiser l'argent, } \\
\text { connaître la vraie amitié). } \\
\text { - Remerciement et clôture divine de } \\
\text { l'interview : « Je suis ici } 24 \text { h par } \\
\text { jour... Tu pourras oublier ce que j'ai } \\
\text { dit/fait, mais pas comment je t'ai fait } \\
\text { sentir avec ces mots. » }\end{array}$ & $\begin{array}{l}\text { Aucune } \\
\text { iconographie } \\
\text { religieuse. } \\
\text { Diptyques de } \\
\text { photos de } \\
\text { banques } \\
\text { d'images : scène } \\
\text { de consultation ; } \\
\text { montre + } \\
\text { cosmos ; } \\
\text { enfants ; dollars } \\
\text { sur le ciel/homme } \\
\text { mourant ; femme } \\
\text { à la plage/ } \\
\text { tombeaux ; main } \\
\text { bébé/adulte ; } \\
\text { couple/famille ; } \\
\text { marteau/manoir ; } \\
\text { femme/couple ; } \\
\text { groupe d'amis. }\end{array}$ & $\begin{array}{l}\text { Transitions } \\
\text { pixellisées. } \\
\text { Reprise d'un } \\
\text { même } \\
\text { pictogramme } \\
\text { (bean figure) } \\
\text { pour les deux } \\
\text { dernières } \\
\text { diapos : un } \\
\text { pictogramme } \\
\text { serrant un } \\
\text { cœur devant } \\
\text { un } \\
\text { pictogramme } \\
\text { auréolé de } \\
\text { cœurs [§12- } \\
\text { 13]. }\end{array}$ & $\begin{array}{l}\text { «Alors, en remerciement à Dieu } \\
\text { pour le temps qu'll nous } \\
\text { accorde, prenez quelques } \\
\text { minutes pour envoyer ce } \\
\text { message à vos meilleurs amis » } \\
\text { "... Affectueusement !! Une } \\
\text { accolade de ton ami ». }\end{array}$ \\
\hline $\begin{array}{l}\text { Profecia } \\
\text { de Maria } \\
\text { en } \\
\text { Fatima.pp } \\
\text { s } \\
\text { [21 diapos] }\end{array}$ & $\begin{array}{l}\text { - Annonce de la « } 3^{e} \text { prophétie de } \\
\text { Fatima - secret de Fatima » que } \\
\text { « l'église catholique, à l'époque, avait } \\
\text { promis de révéler ». } \\
\text { - Revue des Papes de Paul VI à } \\
\text { Benoît XVI [nommé « el Papa } \\
\text { Rachinger ( } \\
\text { ce que la prophec validation de } \\
\text { leurs mandats (durée, orientation...). } \\
\text { - Révélations de la « Sainte Marie » }\end{array}$ & $\begin{array}{l}\text { Fond: Photo } \\
\text { d'une colline avec } \\
\text { des brebis et une } \\
\text { Vierge } \\
\text { superposée } \\
\text { apparue devant } \\
\text { trois petits } \\
\text { bergers à genoux } \\
\text { [dont Lucia]. } \\
\text { - Mention } \\
\text { «Amigos de }\end{array}$ & $\begin{array}{l}\text { Dernière } \\
\text { diapo : } \\
\text { « Sœur Lucia } \\
\text { est décédée } \\
\text { le } 11 \text { février } \\
2005 . \text { Depuis } \\
\text { les années } 20 \\
\text { on avait } \\
\text { prévenu que } \\
\text { cette } \\
\text { prophétie } \\
\text { s'accomplirait }\end{array}$ & $\begin{array}{l}\text { "SVP reproduisez cette feuille } \\
\text { et envoyez ceci à tous ceux que } \\
\text { vous connaissez de sorte que } \\
\text { nous ayons tous l'opportunité de } \\
\text { nous repentir et de survivre. } \\
\text { Nous ne savons pas si ceux qui } \\
\text { sont en train de recevoir ce } \\
\text { message croient ou non en Lui, } \\
\text { mais pensez que si Dieu permet } \\
\text { qu'il arrive à eux c'est par qu'll } \\
\text { les aime quelle que soit leur }\end{array}$ \\
\hline
\end{tabular}


Des Powerpoints en chaînes.

Formes et circulation des diaporamas chrétiens en Amérique latine

\begin{tabular}{|c|c|c|c|c|}
\hline & 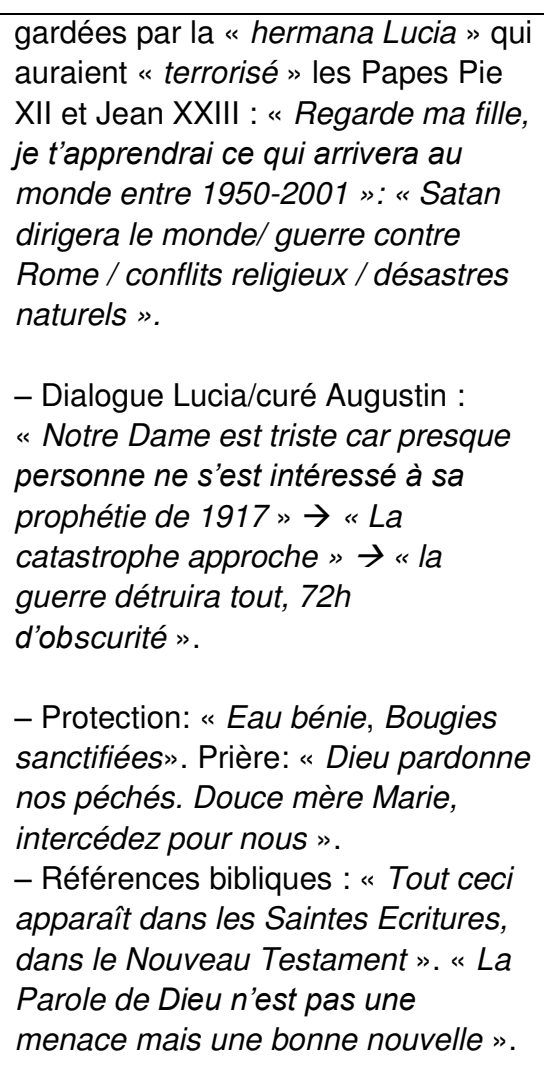 & $\begin{array}{l}\text { Nossa Senhora" } \\
\text { [portugais]. }\end{array}$ & $\begin{array}{l}\text { après sa } \\
\text { mort ». }\end{array}$ & $\begin{array}{l}\text { religion. Si vous ne croyez pas } \\
\text { en ce message, au moins } \\
\text { renvoyez-le à d'autres, cela ne } \\
\text { vous coûte rien, de sorte que } \\
\text { ceux qui croient aient } \\
\text { l'opportunité de décider par eux- } \\
\text { mêmes. } \\
\text { Souvenez-vous du fait que tout } \\
\text { ceci peut être évité si nous } \\
\text { pratiquions les } 10 \\
\text { commandements que le } \\
\text { Seigneur nous a donnés. Si } \\
\text { nous mettions tous ces } 10 \\
\text { choses simples en pratique, } \\
\text { nous pourrions obtenir son } \\
\text { pardon. ". }\end{array}$ \\
\hline $\begin{array}{l}\text { Jesús } \\
\text { conversa } \\
\text { con el } \\
\text { Diablo.pp } \\
\text { s } \\
\text { [21 diapos] }\end{array}$ & $\begin{array}{l}\text { - Récit d'un échange: « Un jour, } \\
\text { Jésus a une conversation avec le } \\
\text { Diable et il demande au diable ce } \\
\text { qu'il est en train de faire avec les } \\
\text { personnes ici sur terre... - Je } \\
\text { m'amuse à leur apprendre à se tuer / } \\
\text { se droguer / se haïr / mentir ... et } \\
\text { après je les achèverai ». } \\
\text { - Tentative de négociation de Jésus : } \\
\text { "Combien veux-tu pour ces } \\
\text { personnes? I... Vraiment ? Elles } \\
\text { sont traitresses, menteuses, } \\
\text { égoïstes, elles ne t'aimeront pas } \\
\text { vraiment et cracheront sur ton } \\
\text { visage... » Relance : «Combien } \\
\text { veux-tu ? .... je veux toutes tes } \\
\text { larmes et tout ton sang / Affaire } \\
\text { conclue ! "Chute : « Et... Jésus } \\
\text { paya le prix de notre liberté ! ». }\end{array}$ & $\begin{array}{l}\text { - Photos et } \\
\text { icônes : } \\
\text { Lever de soleil } \\
\text { sur mer ; Jésus } \\
\text { hors-tombeau + } \\
\text { Diable en } \\
\text { capuche ; Avion } \\
\text { bombardier ; } \\
\text { Personnes } \\
\text { déprimées (SDF } \\
+ \text { la Mort) ; Jésus } \\
\text { priant ; Enfants } \\
\text { morts + tête de } \\
\text { mort + } 11 \\
\text { septembre + } \\
\text { bombe H ; Jésus } \\
\text { auréolé avec } \\
\text { ange et tables, en } \\
\text { sang (cf. Mel } \\
\text { Gibson), crucifié, } \\
\text { portant un } \\
\text { homme, avec } \\
\text { colombe et ange. }\end{array}$ & $\begin{array}{l}\text { - Musique : } \\
\text { "My heart } \\
\text { will go on » } \\
\text { de Céline } \\
\text { Dion en } \\
\text { instrumental. } \\
\text { - Diaporama } \\
\text { auto-animé : } \\
\text { texte surligné } \\
\text { progressivem } \\
\text { ent comme un } \\
\text { karaoké. }\end{array}$ & $\begin{array}{l}\text { “Comme nous oublions Jésus! } \\
\text { Nous croyons tout ce qu'on } \\
\text { nous apprend mais } \\
\text { questionnons tout ce qui vient } \\
\text { de lui... Les gens s'envoient des } \\
\text { blagues par courriel les unes } \\
\text { aux autres à la vitesse de la } \\
\text { lumière! Mais quand le } \\
\text { message porte sur Dieu les } \\
\text { personnes réfléchissent deux } \\
\text { fois avant de l'envoyer et de le } \\
\text { partager avec les autres. Ils } \\
\text { disent tous vouloir être un jour } \\
\text { avec Dieu. Beaucoup disent: Je } \\
\text { crois en Dieu, mais que font-ils } \\
\text { pour Lui?! Parler de Jésus n'est } \\
\text { pas ce que les gens veulent } \\
\text { entendre... ils n'ont recours à } \\
\text { Dieu qu'en cas de gros } \\
\text { problèmes. Quand tu auras fini } \\
\text { de lire ce message l'enverras-tu } \\
\text { à quelqu'un? Qui passera ce } \\
\text { message? Ȧ qui l'enverrez- } \\
\text { vous? Cela dit, n'aie crainte, } \\
\text { mon fils, je serai toujours avec } \\
\text { toi. Même quand tu crois que tu } \\
\text { es seul. Que la paix soit avec }\end{array}$ \\
\hline
\end{tabular}

tic\&société - 9 (1-2), 2015 


\begin{tabular}{|c|c|c|c|c|}
\hline & & & & toi. AMEN. » \\
\hline $\begin{array}{l}\text { El centro } \\
\text { de la } \\
\text { Biblia.pps } \\
\text { [11 diapos] }\end{array}$ & 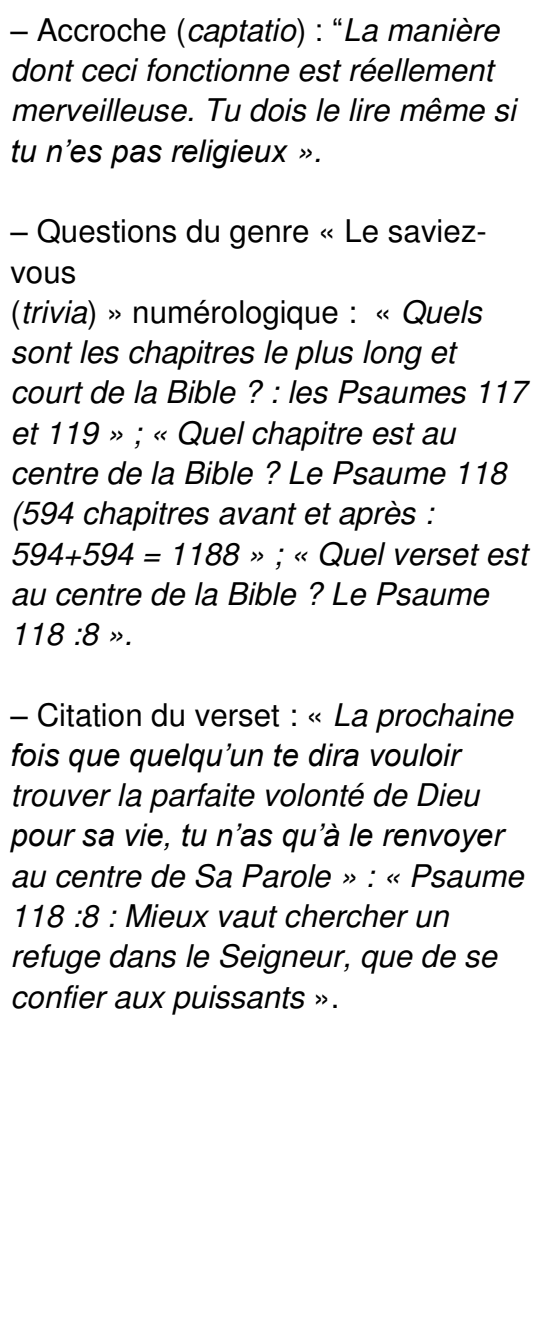 & $\begin{array}{l}\text { - Photos de } \\
\text { paysages tendant } \\
\text { vers le kitsch: Ile } \\
\text { déserte, coucher } \\
\text { de soleil au } \\
\text { désert, nuit au } \\
\text { désert avec } \\
\text { étoiles filantes, } \\
\text { reflets d'arbres } \\
\text { sur un lac, } \\
\text { coucher de soleil } \\
\text { sur une plage, } \\
\text { soleil entre les } \\
\text { montagnes/les } \\
\text { nuages/la mer, } \\
\text { vues en surplomb } \\
\text { sur des chutes } \\
\text { d'eau et sur une } \\
\text { baie } \\
\text { paradisiaque. }\end{array}$ & $\begin{array}{l}\text { - Bruitages et } \\
\text { effets } \\
\text { sonores : } \\
\text { flèche, flash, } \\
\text { machine à } \\
\text { écrire, freins } \\
\text { de voiture. }\end{array}$ & $\begin{array}{l}\text { "N'est-ce pas étrange comme } \\
\text { ça marche, ou est-ce Dieu qui } \\
\text { est au centre de ceci ? Avant de } \\
\text { l'envoyer, puis-je faire une prière } \\
\text { pour toi ? As-tu une minute? } 60 \\
\text { secondes pour Dieu? Tout ce } \\
\text { que tu dois faire est simplement } \\
\text { une petite prière pour la } \\
\text { personne qui t'a envoyé ceci. } \\
\text { "Notre Père bénis mon ami(e) } \\
\text { dans ce dont tu sais qu'il a } \\
\text { besoin aujourd'hui. Et que leur } \\
\text { vie soit pleine de Paix, } \\
\text { Prospérité et Force autant } \\
\text { qu'illelle souhaitera avoir une } \\
\text { relation plus proche avec Toi. } \\
\text { Amen". Envoie ceci à autres } 10 \\
\text { personnes. Dans quelques } \\
\text { heures } 10 \text { personnes auront prié } \\
\text { pour toi, et tu feras qu'une } \\
\text { multitude de personnes prient } \\
\text { les unes pour les autres". Alors } \\
\text { assieds-toi et sens le Pouvoir de } \\
\text { Dieu à l'œuvre dans ta vie, en } \\
\text { faisant les choses que tu sais } \\
\text { qu'll aime. Quand les choses } \\
\text { vont mal rappelle-toi que la foi } \\
\text { ne t'éloigne pas des problèmes } \\
\text { mais te permet de les traverser! } \\
\text { "Quand tu te défais du désir de } \\
\text { contrôler ton avenir, tu atteins le } \\
\text { bonheur ». Que Dieu te } \\
\text { bénisse ». }\end{array}$ \\
\hline
\end{tabular}

Tableau 1. Assemblages sémiologiques des Powerpoints chrétiens. 
Du point de vue analytique, cette mise à plat systématique des exemples qui sont au cœur de notre corpus permet de discerner les procédés techniques, poétiques et stylistiques qui christianisent le cadre du Powerpoint. En ce qui concerne les inscriptions des croyances au sein des diaporamas, des connotations de «religiosité » et de «chrétienté » peuvent se dégager de «messages linguistiques» de plusieurs ordres (Barthes, 1964). D'emblée, les noms mêmes des fichiers souvent calqués sur l' "Objet: » de la chaîne de courriels fonctionnent comme une légende qui requalifie le contenu des diaporamas : "TestamentoDeCristo.pps », «ProfeciaDeMariaenFatima.pps », «EntrevistaConDios.pps » ou «JesusConversaConEIDiablo.pps » suggèrent déjà une « suspension volontaire de l'incrédulité » (suivant la formule de Coleridge) du type ceci n'est pas un Powerpoint, mais un Testament, une Prophétie, une Interview censée mettre en scène un être religieux. L'analyse des messages linguistiques contenus met en évidence deux procédés majeurs qui font qu'un «corps de texte » réparti en moyenne sur 15-20 diapositives puisse dérouler cette promesse conceptuelle, tout en connotant explicitement une «chrétienté » de la présentation (slideshow).

À la base des croyances cristallisées par les contenus textuels des fichiers des chaînes, un procédé primordial consiste à établir une trame narrative comportant un ou plusieurs actants religieux dans laquelle le croyant peut se projeter: " Jésus "voyant que son heure approche", avant de mourir nous dit... "; " J'ai rêvé que j'interviewais Dieu, il m'a dit... »; " la Vierge Marie a parlé à Lucia qui a parlé à l'Eglise qui ne vous a pas tout dit...»; «Un jour, Jésus a une conversation avec le Diable, II lui dit... ". Diverses mises en récit d'un personnage connu des chrétiens et de son dire supposé sont au centre des messages linguistiques des Powerpoint religieux étudiés. II en va de même pour la chaîne «Pourquoi faire confiance à Jésus-Christ? » où Jésus nous demande «Pourquoi t'agites-tu tant avec les problèmes que la vie t'amène? ? et nous invite à lui dire «Jésus je te fais confiance». Sur les territoires des misiones jesuítas, ce procédé fait écho à la tradition ignatienne des «colloques»: exercices spirituels projectifs où sont imaginés des échanges oraux ou « entretiens » d'interlocuteurs transcendants (Loyola, 1672). En ce sens, nous avons affaire à des récits de «colloques»- à leur transcription sous forme de supports Powerpoint - qui ne cessent de renvoyer le croyant latinoaméricain à un archaïsme chrétien fondamental pointé par Michel de Certeau: "Avant la période "moderne", donc jusqu'au XVI-XVII ${ }^{e}$ siècles, cette Écriture (la Bible) parle. Le texte sacré est une voix, il enseigne (premier sens de 
documentum), il est l'advenue d'un "vouloir-dire" de Dieu qui attend du lecteur un "vouloir-entendre" dont dépend l'accès à la vérité » (Certeau, 1980, p.202). Par rapport à ce «vouloirentendre » religieux, ces petits récits de colloques voient leurs fils narratifs favorisés par le fonctionnement linéaire du documentum Powerpoint qui progresse de diapositive en diapositive et attise l'envie de «vouloir avancer» dans la lecture du texte. Or, à partir du moment où la trame narrative est lancée, l'inscription à l'écran de « séquences dialogales » (Kerbrat-Orecchioni, 1990, p.38) riches en tirets de «questionsréponses » semble atténuer le caractère écrit du «colloque », comme pour mieux faire entendre le dire attribué aux "voix » des actants religieux. Par ses formes mêmes, le message linguistique mime une «Parole » en situation, comme s'il la documentait sous forme de Powerpoint.

Dans l'espace culturel latino-américain souvent caractérisé par une prépondérance de «l'oralité » (Martin-Barbero, 1993), ce procédé qui textualise un récit de « voix » transcendantes semble primordial dans la production d'une religiosité sous forme de Powerpoint. Cependant, ce plan linguistique du diaporama peut être complété par des procédés citationnels plus classiques, moins liés à des « citations de voix 》 (Certeau, 1980) qu'à des références Biblio-graphiques. À travers de renvois précis à des extraits de la Bible et des reprises intertextuelles, la trame du Powerpoint tisse ses attaches au Livre saint des chrétiens. En particulier, nous pouvons penser au message linguistique du diaporama " Qu'y a-t-il au centre de la Bible » qui fonctionne comme un déclencheur (teaser) de la recherche du milieu arithmétique des versets et débouche sur une reproduction textuelle du «Psaume 118: 8: Mieux vaut chercher un refuge dans le Seigneur, que se confier aux puissants $»^{5}$. Dans ce même esprit citationnel, le récit de « la $3^{\ominus}$ prophétie de Fatima» se sert d'une liste de tirets pour référencer le «Nuevo Testamento: - S. Lucas (Capítulo 21, versos $5: 11,12: 19,20: 20,29: 33)$ - Carta de S. Paulo (Capítulo 3, 8-14) - El profeta Isaías (cap.40,1:5:9). "6. Une autre chaîne intitulée «Lieux où vécut la Vierge Marie " ne fait

\footnotetext{
4 «La modernité nous parle en Amérique latine de la compénétration et de la complicité entre l'oralité - comme expérience culturelle primaire de la plupart des gens - et la visualité électronique » (Martin-Barbero, 1993, p.40).

${ }^{5}$ Littéralement: “Más vale refugiarse en el Señor que confiar en los poderosos ». Sur Internet, la traduction française la plus usuelle est celle de la Bible de Louis Segond - "Mieux vaut chercher un refuge en l'Éternel que de se confier aux grands »-qui correspond plutôt au verset 9 du même psaume.

Respectivement: "Le Nouveau Testament: -St. Luc (Chapitre 21, versets 5:11, 12:19, 20:20, 29:33) -Lettre de St. Paul (Chapitre 3, 8-14) - Le profeta Isaïe (Chap.40 , 1:5:9)».

${ }^{7}$ En espagnol : «Lugares donde vivió la Virgen María ».
} 
qu'articuler des photos récentes de Bethléem, Nazareth ou Jérusalem à des morceaux choisis des Évangiles. Toutes ces données retissent la diapositive à la Bible et cherchent à lui octroyer une sorte de " crédibilité » institutionnelle. Mais, même lorsque la référence n'est pas explicite, il y a un intertexte «non-avoué » (Barthes, 1981), car les simples évocations linguistiques de "la crèche, la croix ou l'Apocalypse » remettent en mouvement un canon biblique trivialisé.

En ce qui concerne les messages iconiques contenus dans les Powerpoints chrétiens, nous avons affaire à des « images de piété numériques» (Douyère, 2014) qui amplifient les procédés linguistiques que nous venons de décrire. Le matraquage d'images de Jésus - allant jusqu'au gif animé où « II » nous bénit d'un signe de croix -, tout comme l'image de la Vierge, facilitent la projection narrative et fonctionnent comme « des signes de l'immatériel objet de la croyance » (Ibidem). Au fil des diapositives, on observe la dichotomie des visages de Jésus : l'icône auréolée en tunique blanche peut céder sa place au Christ ensanglanté, le Sagrado Corazón de Jesús des dévotions latino-américaines côtoie le casting de la Passion of Christ vue par le réalisateur Mel Gibson. D'un Powerpoint à l'autre, une image récurrente met en scène un Jésus qui sort de son tombeau, comme s'il s'agissait d'illustrer le mystère de sa résurrection.
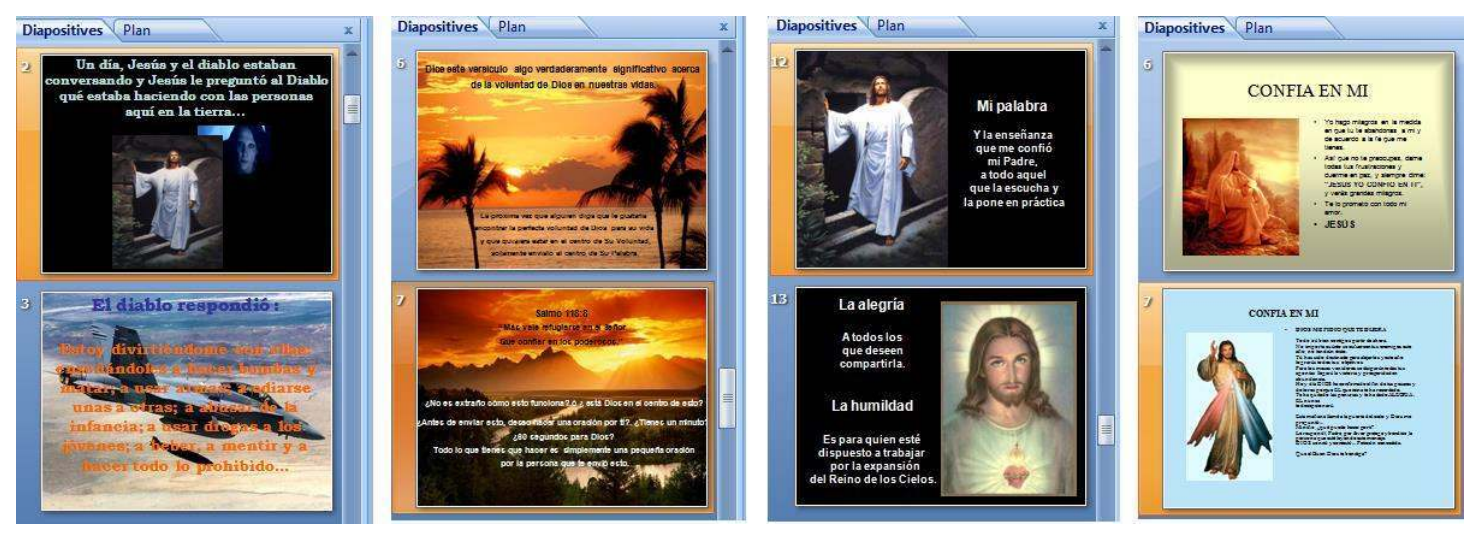

Fig. 2. Messages iconiques de quelques Powerpoints chrétiens.

En retraçant des lignées d' «iconographies religieuses pour usages plébéiens » qui unissent l'Espagne à ses anciennes colonies, Jesús Martín-Barbero rappelle que «les images ont été depuis le Moyen Âge le "livre des pauvres", le texte dans 
lequel les masses ont appris une histoire et une vision du monde imaginées en une clef chrétienne » (2003, p.145). Les Powerpoints chrétiens partent de cette matrice culturelle et la syncrétisent avec d'autres imageries. D'une part, des paysages de cieux, couchers de soleil et des mers paradisiaques semblent connoter la beauté ou l'immensité de la Nature, en même temps que "le côté par lequel elle s'offre au rêve ", ce « kitsch onirique » dont parlait Walter Benjamin (1927). D'autre part, des photos de banques d'images peuvent répondre aux besoins connotatifs du fil textuel dans un spectre qui va des groupes de personnes (amitié, amour, famille) aux scènes dysphoriques (mort, maladie, guerre), en passant par les imageries New Age du cosmos.

Comme l'affirme Flora Delfino, le Powerpoint autorise « une configuration hétérogène qui peut convoquer des aspects liés à des conceptions chrétiennes et aux saints, anges ou vierges, mais aussi des sorcières ou des motifs "orientaux" de la connaissance de soi-même » (Delfino et al., 2006). Cette hétérogénéité s'accroît avec l'ajout d'effets d'animation audiovisuels qui ne connotent nullement la chrétienté et sont censés accompagner le récit et la projection du croyant : fondus filmiques et enchaînements dynamiques qui automatisent le débit des diapositives; musiques de Linda Rondstadt ou de Céline Dion pour connoter un certain pathos; effets sonores (bruitages de flash, machine à écrire ou voiture) et surlignages de karaoké pour dynamiser les «Paroles » affichées comme un " texte nomade » (Souchier, 2000). Ces effets dramaturgiques propres au logiciel de Microsoft complètent l'ambiance d'un diaporama déjà suffisamment christianisé sur le plan scriptoiconique, en même temps qu'ils guident l'attention du croyant et son sens de l'écoute immersive vers le grand moment que constitue la demande de réplication de la « chaîne » religieuse.

Force est de constater que «l'adresse finale au récepteur » reste le principal invariant des chaînes de Powerpoints chrétiens : "Envoie ce courriel à autant de contacts que tu en as », " prenez quelques minutes pour envoyer ce message à vos meilleurs amis", "SVP reproduisez cette feuille et envoyez ceci à tous ceux que vous connaissez »... Du point de vue linguistique, ces adresses sont caractérisées par une « injonction » dont «l'insistance est d'autant plus importante que la désobéissance briserait la chaîne » (Rouquette, 1994, p.41). D'après les exemples analysés, nous sommes loin des injonctions superstitieuses et caricaturales attribuées aux cadenas religiosas du type "faire suivre le fichier = bonne nouvelle; ne pas le faire = mauvaise fortune " (Acevedo Moreno, 2013). Au contraire, deux procédés rhétoriques subtils, susceptibles d'affecter un sujet croyant, nous semblent à 
l'origine de l'efficacité de cette « fonction conative » (Jakobson, 1960) placée en fin de diaporama.

D'une part, il peut être question d'un effet de basculement énonciatif d'une diapositive à l'autre : alors que tout au long du récit il faut "entendre » certains pronoms personnels comme renvoyant à des actants transcendants, une interpellation pronominale ouverte surgit soudainement et intègre le spectateur chrétien à la scène du Powerpoint. «Je vous ai aimé à l'extrême et vous porte dans mon cœur... Envoie ce courriel à autant de contacts que tu en as ». Avec Benveniste (1956), on peut sentir que les voix se confondent : le « Je » (de Jésus) semble momentanément inscrit sur la même scène énonciative que le «tu » (quiconque visionne le Powerpoint) ; le «je » implicite de l'auteur apocryphe bénéficie donc d'un moment d'ambiguïté entre deux diapositives (qui parle maintenant ?) au moment d'adresser son invitation. De même, dans la «conversation de Jésus et le Diable » où ils forment le couple «je-tu», tout à coup surgit un « nous »: «Et... Jésus paya le prix de notre liberté ! ». Pour le sujet croyant, le frisson de la chaîne tient peut-être à cette expérience d'une voix anonyme qui l'embraye sur un plan proche des locuteurs transcendants; à l'écran, il se fait interpeller dans un hic et nunc teinté d'au-delà... et puis, après un «Amen » ou un ornement de clôture, cet écran médiateur disparaît automatiquement. Car c'est le propre du format prédominant «.pps»-Powerpoint Slideshow- que de se fermer tout seul, entretenant ainsi un «culte de la disparition » 8 automatisé qui ne laisse pas au croyant le temps de rationaliser tous ces jeux énonciatifs.

D'autre part, l'effet de chaîne tiendrait à un certain « chantage spirituel » ou « affectif » pour reprendre les mots de nos informateurs latino-américains : alors que les diaporamas regorgent de grandes causes collectives et altruistes (amour universel, prévention de l'apocalypse, leçons pour l'humanité), leurs derniers mouvements mettent toujours les sujets «à contribution » : "En remerciement à Dieu pour le temps qu'll nous accorde, prenez quelques minutes pour envoyer ce message à vos meilleurs amis »; "Les gens s'envoient des blagues par courriel les unes aux autres à la vitesse de la lumière! Mais quand le message porte sur Dieu les personnes réfléchissent deux fois avant de l'envoyer et de le partager avec les autres. Quand tu auras fini de lire ce message l'enverras-tu à quelqu'un ? "; "Envoie ceci à 10 autres personnes. Dans quelques heures 10 personnes auront prié pour toi, et tu feras

\footnotetext{
${ }^{8}$ En écho à la formule « culte de l'apparition » de Pierre d'Huy (2007), commentée par David Douyère (2011).
} 
qu'une multitude de personnes prient les unes pour les autres ». En ce sens, l'injonction à « faire suivre » exploite les disproportions existantes entre la grande cause éternelle et le petit clic mondain ; elle teste ainsi la propension des sujets à se sentir reconnaissants ou ingrats, altruistes ou égoïstes, fiers ou coupables face au choix proposé de devenir (ou non) un maillon de la chaîne. Dans le cas du diaporama "Pourquoi faire confiance à Jésus-Christ ? » on lit ainsi : "Si tu n'envoies pas ce fichier tu n'auras pas de mauvaise fortune, car la chance n'est pas ordonnée par DIEU ; tu auras simplement manqué de partager quelque chose de "réellement important" avec les autres ». Au moment où la dernière diapositive « disparaît », l'éventuel dilemme de l'envoi du fichier confronte donc le croyant aux obligations d'une logique du don (Mauss, 1924) distordue par une stéréotypique culpabilité judéo-chrétienne : il est question de faire croire que l'offrande rituelle du clic préviendrait les croyants d'être en dette. De sorte que l'adresse finale pourrait être reformulée comme suit : «En refusant de faire suivre cette chaîne, vous entraîneriez une déperdition d'échanges symboliques réciproques (bénédiction, pardon, protection, gratitude) censés avoir lieu entre l'œil de Dieu, le regard de vos "connaissances" et votre for intérieur. "

\section{Points de contact, points de pouvoir : des croyances latino-américaines mises en tension}

Comme nous l'avons vu, les chaînes de Powerpoint entremêlent pour le sujet croyant ce qui relève d'une "identification horizontale » - aux «connaissances », aux groupes de "contacts»- et ce qui renvoie à une « identification verticale » - à Dieu, à Jésus, à la Vierge et à d'autres pouvoirs transcendants (Ollivier, 2007, p.131). II semble impossible de dissocier ces deux pôles d'identification pour isoler celui qui serait l'objet du croire : l'affirmation du collectif de «contacts» est intimement liée à la révérence envers le pouvoir symbolique des diapositives. D'où la nécessité d'interroger non seulement la manière dont la religion vient au Powerpoint, mais aussi la dimension sociale du geste de « faire suivre » et les pouvoirs qu'il affirme en tant qu'acte de croyance inscrit dans une mondialisation culturelle.

Michel de Certeau (1980, p.260) proposait d'entendre par " "croyance" non pas l'objet du croire (un dogme, un programme, etc.), mais l'investissement des sujets dans une proposition, l'acte de l'énoncer en la tenant pour vraie autrement dit une modalité de l'affirmation et non pas son contenu ». Ce distinguo est pertinent, car, d'un point de vue latino-américain, il semble aisé de disqualifier le contenu de ces 
chaînes chrétiennes comme du spam ou de folkloriser la religion ré-imaginée sous forme de diaporama. La plupart des discours d'internautes latino-américains sur les cadenas religiosas s'en tiendraient souvent à des niveaux superficiels de jugement : « [ce] "gribouillage cybernétique" qu'est devenu la diffusion de la foi par des chaînes » (Totouy, 2011), ou encore " [...] la véracité n'est pas démontrée pour les "témoignages" d'êtres divins, lesquels ne dépassent jamais le stade d'anecdotes. Si ces croyances n'ont pas eu de validité dans le monde réel, pourquoi en auraient-elles dans le monde numérique ? » (Aranda, 2015). Or, le "fait que des sujets s'investissent " au quotidien dans le credo de leur circulation (ou dans leur contestation) à l'échelle du continent nous invite à aborder les implications sociopolitiques du phénomène des chaînes religieuses. Si l'on accepte la proposition de Certeau (1974, p.30) selon laquelle « une société résulte de la réponse que chacun donne à la question de son rapport à la vérité et de son rapport aux autres ", ces chaînes nous donnent également à lire des enjeux de pouvoir qui dépassent l'écran et reflètent deux grands lignes de tension qui animent le paysage des croyances au sein des sociétés latino-américaines contemporaines.

La première ligne de tension que révèlent ces chaînes concerne la transformation du paysage des croyances dans un territoire étiqueté comme «traditionnellement catholique », mais qui le serait de moins en moins au vu de l'emprise sociale croissante d'une myriade d' "iglesias cristianas, evangélicas, carismáticas, protestantes". Dans son ouvrage L'Amérique latine devient-elle protestante?, l'anthropologue américain David Stoll refusait déjà toute quantification du phénomène en rappelant que «la grande majorité des latino-américains sont catholiques juste de nom », tandis que les «protestants » tendent a contrario à être assimilés à de « vrais protestants » et que les "églises évangéliques » accordent une importance politique au fait de «maximiser leurs taux de croissance » (Stoll, 1993, p.19). Face à cette perte de puissance politique et démographique de l'Église romaine dans la région, on pourrait dire que ces chaînes contribuent à un brouillage des frontières entre catholiques et évangéliques, car leurs Powerpoints religieux sont a priori indéterminés et, pour reprendre l'expression de Michel-Louis Rouquette (1994, p.20), "S'inscrivent dans « des procédés de communication et de mobilisation "parallèles", qui traduisent l'effet d'initiatives en marge de l'institution officielle ecclésiastique ». Cependant, au vu de l'ampleur sociale de leur circulation, les chaînes sont aussi des points de contact de diverses populations «chrétiennes", au sein desquelles peuvent se cliver les 
postures des soi-disant « vrais catholiques» ou « vrais protestants ».

Dans ces clivages entre "chrétiens " latino-américains, plusieurs articles sur les cadenas religiosas reproduisent le texte d'une chaîne ouvertement catholique - signée par un prêtre, le Padre Adolfo Franco- qui est paradoxalement destinée à combattre " ces agaçantes chaînes » :

"Ces chaînes sont une ABERRATION qui va à l'encontre de la vraie foi ; elles veulent manipuler la Providence de Dieu qui, étant divine, agit librement et n'est pas assujettie à des conditions comme « envoie ceci à 10 personnes » [...]. Sans mettre en doute la bonne foi des expéditeurs, on peut apprécier que dans leur majorité ce sont des personnes qui manquent de formation chrétienne, puisque celle-ci, quand on la possède, permet d'aimer Dieu et ses semblables sans aucune condition $»$.

Sur ce même argument de la « formation chrétienne », mais du côté de ceux qui se veulent de "vrais évangéliques", une informatrice nous racontait que son « pasteur » recommandait de " ne pas envoyer des courriels avec l'image du Seigneur, car c'est de l'idolâtrie ». À la lumière de son témoignage, la faible représentation de la Vierge et des saints dans notre corpus, ainsi que la neutralisation iconographique des Powerpoints où le corps de Jésus cède sa place à des paysages et autres imageries "naturelles », prennent un sens autre qu'esthétique et deviennent une condition sémio-politique d'acceptabilité du message par certaines obédiences protestantes. Entre ces deux postures clivantes, on peut ajouter I'hypothèse lucide de Moisés Sbardelotto (2014) qui relie la « circulation » accrue de ces formes numériques au fait qu'elles prennent leurs distances par rapport aux «pouvoirs et contrôles » stricts des autorités religieuses et offrent aux internautes croyants l'occasion d'une « échappée doctrinale ».

Parmi ces autorités qu'affirment et relaient les croyants, une deuxième ligne de tension plus diffuse révélée par ces chaînes nous renvoie à une géographie des représentations hégémoniques au sein du monde religieux. Au sujet du Powerpoint chrétien, Douyère (2011) écrit: « II n'est pas étonnant que le religieux s'y insère, suivant, sans doute, une mode américaine ». Si on creuse cette piste, il convient d'observer qu'au-delà des logiciels de Microsoft et des

\footnotetext{
${ }^{9}$ Texte également reproduit - et apparemment authentifié - sur le blog catholique de Carlo Santostefano : <http://carlossantostefano.blogspot.com/2014/01/cadenas-deoracion-padre-adolfo-franco.html>, dernière consultation le 11 août 2015.
} 
industries d'internet californiennes, la dynamique d'affirmation des chaînes de Powerpoints par les croyants reterritorialise dans l'Amérique latine une religiosité relativement peu autochtone et à maints égards subalterne de celle de l'Amérique du Nord.

Comme dans d'autres espaces médiatiques, les croyants «latinos» se retrouvent ainsi à adorer des imageries Powerpoint dont les phénotypes et les coups de crayon correspondent à des canons made in the USA: l'image récurrente du Jésus qui sort de son tombeau se trouve être une reproduction du tableau «He Lives» de l'américain Simon Dewey, commercialisé par l'Altus Fine Art Gallery ${ }^{10}$. Sur le plan linguistique, il n'est semble-t-il pas surprenant que pour répondre à la question «Où va-t-on quand on décède ? 》 les allusions à Dieu, à l'église et les images d'anges dialoguent avec la promotion du livre On Life after Death du Dr. Elizabeth Kübler-Ross. En début et fin du diaporama « Donne du temps à Dieu » un lien pointe vers www.tommyswindow.com, un site américain qui centralise des Powerpoints présentés comme des «Motivational and Inspirational Messages » et demande à être soutenu par des dons en espèces.

Quelques indices supplémentaires sur la trajectoire géographique de ces chaînes nous suggèrent que l'Amérique hispanique serait une grande importatrice de diaporamas en VO portugaise confirmant ainsi le Brésil comme puissance religieuse continentale. D'une part, l'observation minutieuse des modèles (templates) des diaporamas nous révèle l'emploi d'un gabarit nommé « design padrão » correspondant à une version portugaise du logiciel Powerpoint. D'autre part, certaines fautes d'orthographe s'avèrent être des scories lusophones et - sous couvert de «Prophétie de Fatima»- les «Amis de Nossa Senhora » vont jusqu'à décrire un paysage de croyances qui rappelle le «marché » brésilien des religions : "Nombre de ceux qui se sont égarés (afastados) retourneront aux bras de l'Église de Jésus-Christ. [...] Tous les religieux, les protestants, les spirites, les musulmans, les bouddhistes et les juifs ».

10 Altus Fine Art Gallery - Simo Dewey - Product Catalog: <http://www.altusfineart.com/home/afa/smartlist 101/he lives.html>, $\quad$ dernière consultation le 11 août 2015 (voir fig. 2). 


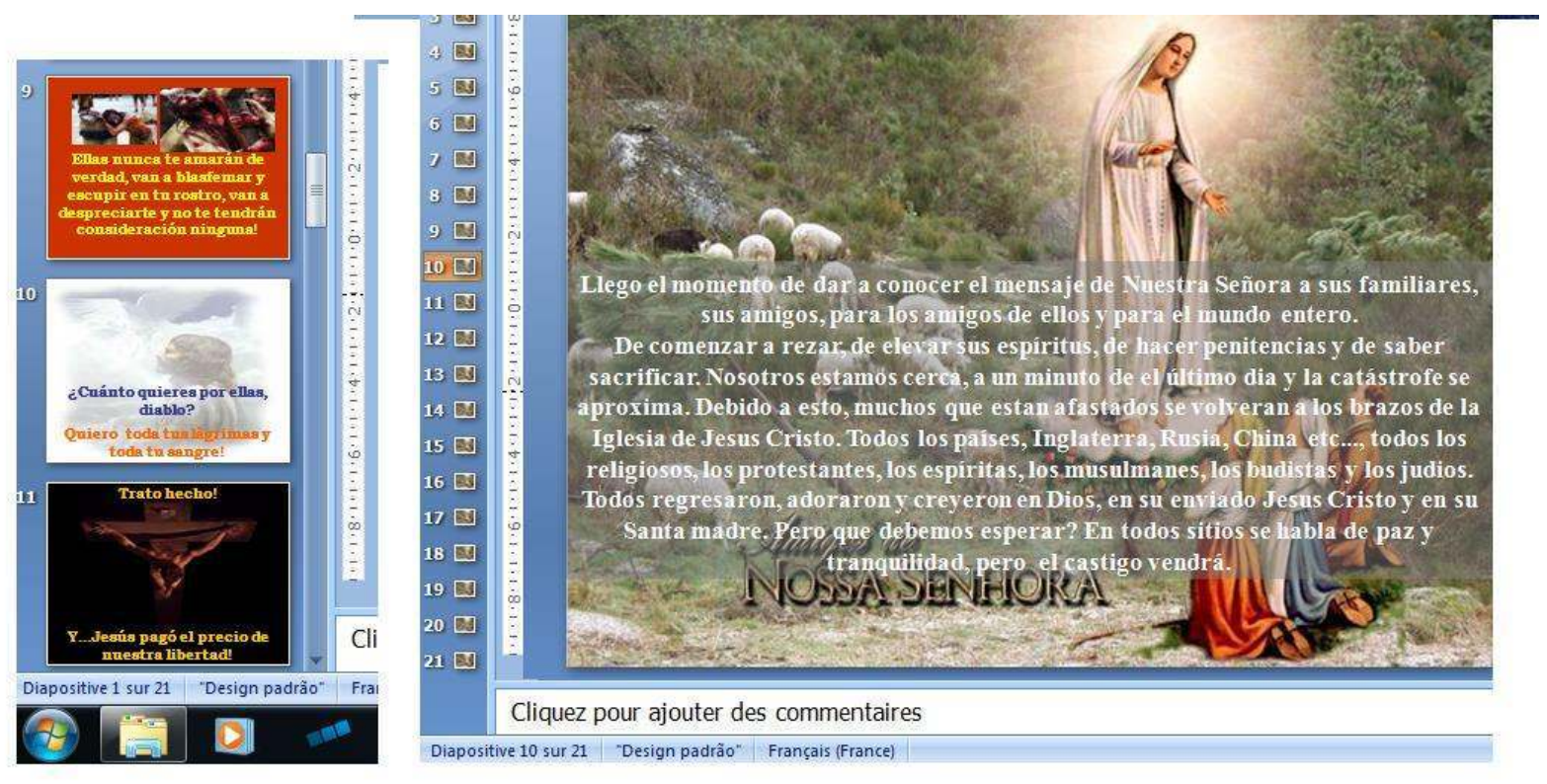

Fig. 3 : Empreintes brésiliennes sur deux Powerpoints chrétiens.

\section{Conclusion}

À la lumière de ces ouvertures qui transcendent les cadres des écrans, les chaînes latino-américaines de Powerpoints chrétiens inscrivent les croyances dans un mouvement de plus en plus "évident à échelle globale " d' " oscillation entre Tradition et Traduction ", par lequel les identités culturelles se trouvent confrontées aux tensions d'une "transition entre différentes positions » (Hall, 1992, p.310). Sans prétendre hypertrophier les tenants et aboutissants d'une lecture " métissée " du religieux, l'étude de ces cadenas religiosas aux apparences anecdotiques remet en perspective quelques complexités contemporaines des supports et des gestes liés au « faire croire ».

Comme nous l'avons vu, le halo "mystérieux " qui entoure ces chaînes tient en premier lieu au fait que leurs auteurs originaux ne sont pas identifiables, ce qui laisse la main libre à l'expéditeur pour se les approprier à son tour et les réexpédier en un clic à d'autres contacts jugés croyants. En deuxième lieu, du côté des pièces jointes qui circulent par ce biais, nous avons pu identifier les principaux procédés par lesquels une présentation Powerpoint prétend accéder au statut d'objet du croire chrétien : titres valorisant le contenu du fichier, mises en scène d'êtres religieux, retissages de textes bibliques et 
d'iconographies pieuses caractérisent ces objets formellement. De même, il convient de rappeler ici à quel point les effets dramaturgiques et énonciatifs des diaporamas jouent un rôle primordial pour enjoindre le sujet croyant à transmettre ce fichier, souvent sur la base d'obligations symboliques de partage et de don. Enfin, ces pratiques par lesquelles tant d'internautes latino-américains deviennent les «maillons » de ces chaînes cristallisent également des enjeux de pouvoir: elles semblent parfois brouiller le temps d'un mail les frontières entre les églises romaines et évangéliques; elles affichent souvent une certaine hégémonie de représentations aux accents américains ou brésiliens sur cette aire d'échanges religieux.

À la croisée de la perpétuation par mail d'une tradition apocryphe de mise en circulation de croyances chrétiennes sous forme de chaînes, de la traduction des messages d'une chrétienté nécessairement syncrétisée avec les langages et les rhétoriques du format Powerpoint, et de la transition de l'Amérique latine dite "catholique » vers des paysages de croyances globalement poreux, ces diaporamas enchaînent d'ordinaire les scènes héritées d'une histoire culturelle aux circuits informels des échanges en réseau.

\section{Références bibliographiques}

ACEVEDO MORENO M. C., 2013, «Los e-mails espirituales: cadenas de fe o falsedad », Suite101, 3 juillet, $<$ http://suite101.net/article/los-e-mails-espirituales-cadenas-defe-o-falsedad-a67180>, dernière consultation le 11 août 2015.

ARANDA Y., 2015, « Las Cadenas Religiosas del Internet », Sabiduría Herética, juillet <https://sabiduriaheretica.wordpress.com/2015/07/11/lascadenas-religiosas-del-internet/>, dernière consultation le 2 septembre 2015.

BARTHES R., 1964, "Rhétorique de l'image », Communications, $\mathrm{n}^{\circ} 4$, pp.40-51.

BARTHES R., 1973, Le plaisir du texte, Paris, Le Seuil.

BENJAMIN W., 1927 [2000], «Kitsch onirique », dans CEuvres II, Paris, Folio.

BENVENISTE E., 1956 [1966], «La nature des pronoms », dans Problèmes de Linguistique Générale I. Paris, Gallimard. 
BONNET S. et A. DELESTRE, 1984, "Les chaînes magiques ", Revue des sciences sociales de la France de l'Est, n ${ }^{\circ} 13-13$, pp.383-402.

CERTEAU M. de, 1974, La culture au pluriel, Paris, Le Seuil.

CERTEAU M. de, 1980, L'invention du quotidien, Tome 1. Arts de faire, Paris, Gallimard.

DELFINO KRAFT F., M.I. PALLEIRO et P. PARENTE, 2006, «La construcción discursiva de la creencia desde el Folclore: relatos orales, narrativas OVNI, mails en cadena ", RIF, $\mathrm{n}^{\circ 21}$, pp.102-114.

DESPRÉS-LONNET M., A. GENTES, E. MOREAU et C. ROQUES, 2003, "Le couple dispositif/pratique dans les échanges interpersonnes ", dans E. SOUCHIER, Y. JEANNERET et J. LE MAREC (dir), Lire, écrire, récrire: Objets, signes et pratiques des médias informatisés, Paris, BPI, pp.161-232.

DOUYĖRE D., 2011, «La prière assistée par ordinateur », Médium, n²7, pp.140-154.

DOUYÈRE D, 2014, “L'image de piété chrétienne, objetsupport de la croyance ? Communiquer la foi par l'image, de l'imprimé au numérique ", Recherches en communication, n³8, pp.29-46.

GIL G., 2006, "La Revolución de la fé », Prensa Libre, 6 août, <http://ow.ly/LbZX7>, dernière consultation le 11 août 2015.

HALL S., 1992, "The Question of Cultural Identity ", dans S. HALL, D. HELD et T. McGREW (dir), Modernity and its Futures, Cambridge, Polity.

HOWARD R. G., 2008, "The Vernacular Web of Participatory Media ", Critical Studies in Media Communication, vol.25, n5, pp.490-513.

HUY P. d', "PowerPoint, la rhétorique universelle ", Medium, n¹1, 2007, pp.12-25.

LOYOLA I. de, 1672, Les exercices spirituels, Anvers, Cnobbert.

MARTÍN-BARBERO J., 2003, De los medios a las mediaciones, Bogotá, CAB.

MARTÍN-BARBERO J., 1993, «La comunicación, centro de la modernidad. Una peculiar relación en América Latina ", Telos, Cuadernos de la comunicación, tecnología y sociedad, n³6, pp.39-46. 
JAKOBSON R., 1960, "Linquistique et poétique », dans Essais de linguistique générale, Paris, Minuit, pp.213-222.

JEANNERET Y., 2008, Penser la trivialité, La vie triviale des êtres culturels, Paris, Hermès Lavoisier.

JENKINS H., 2009, «If it doesn't spread, its dead» <http://henryjenkins.org/>, dernière consultation le 11 août 2015.

LAMBERT F., 2013, Je sais bien mais quand même. Essai pour une sémiotique des images et de la croyance, Paris/Le Havre, Non-Standard.

KERBRAT-ORECCHIONI C., 1990, Les interactions verbales, tome I, Paris, Armand Colin.

MAUSS M., 1994 (2001), «Essai sur le don », dans Sociologie et anthropologie, Paris, PUF.

OLLIVIER B., 2007, Identité et identification : sens, mots et techniques, Paris, Lavoisier.

PERRIAULT J., 1989, La logique de l'usage, essai sur les machines à communiquer, Paris, L'Harmattan.

ROUQUETTE M.-L., 1994, Chaînes magiques. Les maillons de l'appartenance, Neuchâtel, Delachaux \& Niestlé.

SBARDELOTTO M., 2014, «La reconstrucción de lo "religioso" en la circulación en redes socio-digitales », La Trama de la Comunicación, vol.18, pp.151-170.

SOUCHIER E., 2000, «De la lettrure à l'écran. Vers une écriture sans mémoire ? », Texte, $\mathrm{n}$ ²5-26, pp.47-68.

STOLL D, 1993, ¿América Latina se vuelve protestante? Las políticas del crecimiento evangélico, Quito, Planeta.

TARDY C. et Y. JEANNERET (dir), 2007, L'écriture des médias informatisés. Espaces de pratiques, Paris, Lavoisier.

TOTOUY, 2011, «Las cadenas religiosas», Taringa, novembre, $\quad<$ http://www.taringa.net/posts/apuntes-ymonografias/9659542/Las-cadenas-religiosas.html>, dernière consultation le 11 août 2015.

VANARSDALE D., 1998, Chain Letter Evolution. $<$ http://www.silcom.com/ barnowl/chain-letter/evolution.html>, dernière consultation le 11 août 2015. 